

\title{
Combining DFT and CALPHAD for the development of on-lattice interaction models: The case of Fe-Ni system
}

Yimi Wang, Kangming Li, Frédéric Soisson, Charlotte Becquart

\section{To cite this version:}

Yimi Wang, Kangming Li, Frédéric Soisson, Charlotte Becquart. Combining DFT and CALPHAD for the development of on-lattice interaction models: The case of Fe-Ni system. Physical Review Materials, 2020, Physical Review Materials, 4 (11), pp.113801. 10.1103/physrevmaterials.4.113801. hal-03007250

\section{HAL Id: hal-03007250 \\ https://hal.univ-lille.fr/hal-03007250}

Submitted on 16 Nov 2020

HAL is a multi-disciplinary open access archive for the deposit and dissemination of scientific research documents, whether they are published or not. The documents may come from teaching and research institutions in France or abroad, or from public or private research centers.
L'archive ouverte pluridisciplinaire HAL, est destinée au dépôt et à la diffusion de documents scientifiques de niveau recherche, publiés ou non, émanant des établissements d'enseignement et de recherche français ou étrangers, des laboratoires publics ou privés. 


\title{
Combining DFT and CALPHAD for the development of on-lattice interaction models: the case of Fe-Ni system
}

\author{
Yimi Wang, ${ }^{*}$ Kangming $\mathrm{Li}^{\dagger}{ }^{\dagger}$ and Frédéric Soisson ${ }^{\ddagger}$ \\ Université Paris-Saclay, CEA, Service de Recherches \\ de Métallurgie Physique, 91191, Gif-sur-Yvette, France \\ Charlotte S. Becquart $\$$ \\ Univ. Lille, CNRS, INRAE, Centrale Lille, UMR 820\%, UMET, \\ Unité Matériaux et Transformations, F 59000, Lille, France
}

(Dated: November 9, 2020) 


\begin{abstract}
We present a model of pair interactions on rigid lattice to study the thermodynamic properties of iron-nickel alloys. The pair interactions are fitted at $0 \mathrm{~K}$ on ab initio calculations of formation enthalpies of ordered and disordered (special quasi-random) structures. They are also systematically fitted on the Gibbs free energy of the $\gamma$ Fe-Ni solid solution as described in a CALPHAD (CALculation of PHAse Diagrams) study by Cacciamani et al. This allows the effects of finite temperature, especially those of magnetic transitions, to be accurately described. We show that the ab initio and CALPHAD data for the $\gamma$ solid solution and for the $\mathrm{FeNi}_{3}-\mathrm{L}_{2}$ ordered phase can be well reproduced, in a large domain of composition and temperature, using first and second neighbor pair interactions which depend on temperature and local alloy composition. The procedure makes it possible to distinguish and separately compare magnetic, chemical and configuration enthalpies and entropies. We discuss the remaining differences between the pair interaction model and CALPHAD, which are mainly due to the treatment of the short-range order and configurational entropy of the solid solution. The FCC phase diagram of the Fe-Ni system is determined by Monte Carlo simulations in the semi-grand canonical ensemble and is compared with experimental studies and other models. We especially discuss the stability of the FeNi-L1 $1_{0}$ phase at low temperature.
\end{abstract}

\title{
I. INTRODUCTION
}

Atomistic Monte Carlo simulations of phase transformation kinetics in metallic alloys (precipitation, ordering, etc. [1-13]) require interaction models that allow a precise description of the thermodynamic and kinetic properties of the materials, while remaining simple enough to model systems of a few million atoms over long periods of time (their evolution being controlled by thermally activated diffusion mechanisms). Models using effective interactions on rigid lattices - although limited to coherent problems - are among the most widely used and have become more reliable since they are systematically fitted to ab initio calculations of materials properties at $0 \mathrm{~K}$ (such as the formation energies of ordered phases or special quasi-random structures, point defect formation and migration energies, etc.) $[5,7,13]$.

\footnotetext{
* yimi.wang@cea.fr

$\dagger$ kangming.li@cea.fr

¥ frederic.soisson@cea.fr

$\S$ charlotte.becquart@univ-lille.fr
} 
Evaluating non-zero temperature effects from ab initio methods is more difficult: calculations of vibration entropy, for example, are in principle possible but are computationally expensive and are usually limited to simple systems (e.g. pure metals, perfectly ordered phases, or dilute alloys). Modeling the effect of magnetic transitions and magnetic disorder - especially important in iron based alloys - is also very challenging. Alternatively, the temperature dependence of pair interactions can be adjusted on experimental data, for example on phase diagrams, but these adjustments are often made on a case-by-case basis. We propose here a new approach to systematically fit a pair interaction model both on ab initio calculations at $0 \mathrm{~K}$ and, for high temperatures, on a CALPHAD-type model. CALPHAD models provide an accurate description of the Gibbs free energies of the different phases of an alloy, based on empirical thermodynamic models fitted (mainly) on large numbers of experimental measurements. They also provide a specific description of some important contributions (e.g. magnetic contributions in iron-based alloys). The objective of this paper is to show how to establish a term-to-term correspondence between the empirical models used in CALPHAD and the effective interactions of a lattice model; to show the improvements that this brings to the description of a particular alloy; but also to discuss the limits of such a correspondence.

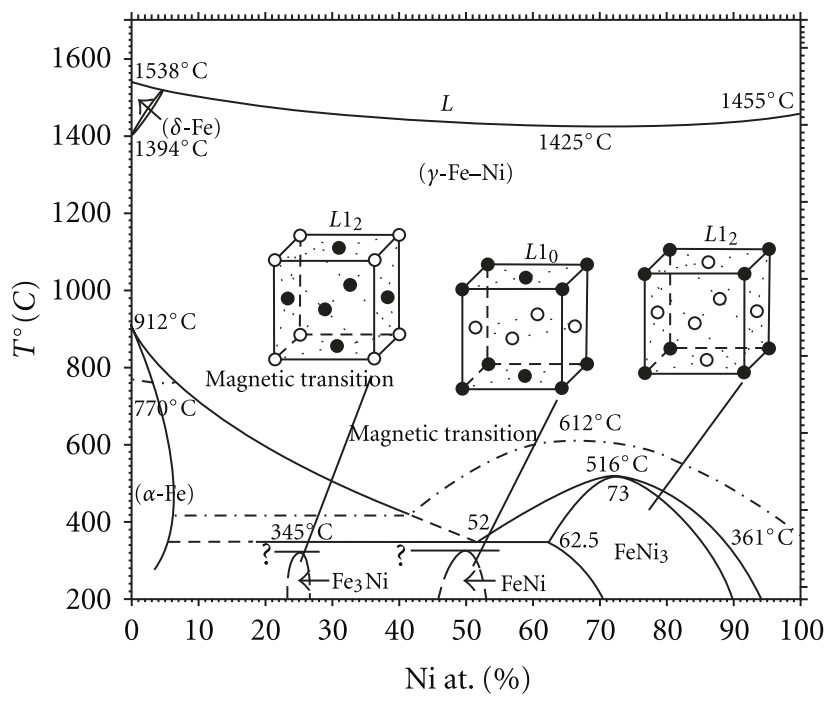

FIG. 1. The phase diagram of the Fe-Ni system (from [14] and [15])

We apply this approach to Fe-Ni alloys with a face-centered cubic structure (FCC). Recent ab initio $[16,17]$ and CALPHAD [18, 19] studies are available for this system. The thermodynamic properties of Fe-Ni alloys have been much studied, because of their industrial 
interest and because they still raise unresolved questions. The phase diagram of the Fe-Ni system (Fig. 1) is well known at temperatures above $400^{\circ} \mathrm{C}$ [20]: the FCC solid solution $\gamma$ is stable over the whole composition range and over a wide temperature range. The body centered cubic (BCC) solid solution $\alpha$ is stable below $912^{\circ} \mathrm{C}$, and only with nickel contents below approximately 10\%. A two-phase domain $\alpha-\gamma$ is observed in iron-rich alloys, and an ordered $\mathrm{FeNi}_{3}$ phase (with the $\mathrm{L}_{2}$ structure) is formed below $516^{\circ} \mathrm{C}$.

At lower temperatures, the phase diagram is - as usual - more difficult to establish, because of slow diffusion processes. However, irradiation experiments [21-23], observations of meteorites $[15,24]$, and ab initio studies $[16,17]$ suggest that other ordered FCC phases may be stable or metastable (notably the FeNi phase of $\mathrm{L}_{0}$ structure and the $\mathrm{Fe}_{3} \mathrm{Ni}$ phase of $\mathrm{L1}_{2}$ structure). Many CALPHAD studies have sought to complement the Fe-Ni phase diagram by extrapolating at low temperatures the empirical thermodynamic models fitted to experiments at high temperatures. This is especially difficult in the case of Fe-Ni, and as a result, the proposed phase diagrams show significant differences [15, 18, 20, 25]. A particular difficulty of this system is that the experimental data are obtained essentially in paramagnetic phases, whereas the ordered phases are ferromagnetic. Magnetic contributions are indeed taken into account in CALPHAD approaches, but again by empirical models using experimental data and still under discussion [25].

Atomistic models combining the information from experiments and first principle methods may provide additional insight on these issues. A few rigid lattice interaction models, fitted to ab initio calculations, have been developed for Fe-Ni alloys: Mohri et al. [26] proposed a cluster expansion to study the stability of the ordered compound FeNi-L1 $1_{0}$ in the framework of a CVM approximation: they found an ordering temperature of $483 \mathrm{~K}$ (taking into account the vibration entropy, which lowers it by about $40 \mathrm{~K}$ ). But they did not study the $\mathrm{FeNi}_{3}$ phase, nor the effect of the ferro-to-paramagnetic transition. Effective interactions models including an explicit description of the magnetic moments have been proposed for Fe-Ni alloys, using Ising [27-30] or Heisenberg [30, 31] models for the magnetic interactions and parameters fitted to the experimental transition temperatures. Similar magnetic models have been also used in phase-field simulations of ordering and precipitation of the $\mathrm{FeNi}_{3}$ phase [32]. More recently Lavrentiev, Wrobel et al. [33, 34] developed a Magnetic Cluster Expansion (MCE), based on a Heisenberg-Landau Hamiltonian, fitted to ab initio calculations. Its properties have been studied by Monte Carlo methods, but the combined equilibration of 
the chemical and magnetic configurations is very costly in computational time, and the complete phase diagram of the MCE model of Fe-Ni remains to be established.

In the present paper, we propose a pair interaction model (PIM) based on a rigid lattice approximation, aiming at modelling the Fe-Ni system. The model does not describe explicitly the magnetic moments, nor the lattice relaxations, which makes it faster to process in Monte Carlo simulations. It only involves pair interactions between atoms that depends both on the local composition and temperature. Finite temperature effects of magnetic transitions or of lattice vibrations (harmonic and non-harmonic), on the energetic proprieties are taken into account through these dependencies.

The outline of this article is as follows: section II is devoted to the thermodynamic models of FCC phases in the Fe-Ni system. We briefly recall the Gibbs free energy models used in the CALPHAD approaches, focusing on the recent study of Cacciamani et al. [18] (II A); then the available ab initio results on the properties of Fe-Ni alloys (in particular those of K. Li and C.-C. Fu [17]) (II B). We then show how to reproduce these results with the PIM in section (II C). In section III, we use Monte Carlo simulations in the semi-canonical grand ensemble, to measure the Gibbs free energies of the FCC alloys, and to build the FCC phase diagram.

\section{THERMODYNAMIC MODELS}

Our PIM is built using both a CALPHAD study and ab initio calculations. We therefore recall the main information provided by these two approaches before to explain how it can be reproduced with effective interactions on a rigid lattice.

\section{A. CALPHAD Models}

Several CALPHAD-type studies have been proposed for the Fe-Ni system: the most

recent are those of Cacciamani et al. [18] and Ohnuma et al. [19] (for older ones, see the reviews in refs. $[15,20])$. Within the CALPHAD framework, a Gibbs free energy model can be defined for each of the phases to be considered (e.g. in the Fe-Ni system, the $\alpha$ and $\gamma$ solid solutions and the different ordered phases). This gives great flexibility to fit the parameters to the experimental data. We will fit our PIM parameters to the study by Cacciamani 
et al. [18] (which will be hereafter simply referred to as the "CALPHAD model" or even as "CALPHAD"), but the following presentation and procedure could easily be adapted to other CALPHAD studies.

\section{The $\gamma$ solid solution}

The Gibbs free energy per atom of the solid solution $\gamma$ (FCC) $\mathrm{Fe}_{1-x} \mathrm{Ni}_{x}$ is:

$$
G^{\gamma}=G_{r e f}^{\gamma}+G_{e x}^{\gamma}+G_{m a g}^{\gamma}+G_{i d}^{\gamma}
$$

This is the total Gibbs free energy, including the entropy of configuration. (Note that in CALPHAD one rather uses molar energies, in $\mathrm{J} . \mathrm{mol}^{-1}$. We convert them in energies per atom, in $\mathrm{eV})$.

$G_{r e f}^{\gamma}$ is the non-magnetic contribution of pure metals:

$$
G_{r e f}^{\gamma}=(1-x) G_{F e}^{\gamma}-x G_{N i}^{\gamma}
$$

where $G_{F e}^{\gamma}$ and $G_{N i}^{\gamma}$ are the Gibbs free energy of pure Fe and pure Ni.

$G_{e x}^{\gamma}$ is the excess Gibbs free energy, written as a sum of Redlich-Kister polynomials:

$$
G_{e x}^{\gamma}=x(1-x) \sum_{i} L_{i}^{\gamma}(T)(1-2 x)^{i}
$$

(from $i=0$ to 2 in [18])

$G_{m a g}^{\gamma}$ is the magnetic contribution:

$$
G_{m a g}^{\gamma}=-k_{B} T f(\tau) \ln (\beta(x)+1)
$$

$\tau=T / T_{c}(x)$, where $T_{c}(x)$ is the Curie temperature, $\beta(x)$ the average magnetic moment of the

$\gamma$ solid solution and $f(\tau)$ is a polynomial function of the reduced temperature. $T_{c}(x)$ and $\beta(x)$ are also given by Redlich-Kister polynomials of the composition $x$, fitted to experimental measurements. Different expressions and values have been proposed [25], those used by Cacciamani et al. are given in [18].

$G_{i d}^{\gamma}$ corresponds to an ideal entropy of configuration:

$$
G_{i d}^{\gamma}=-T S_{i d}^{\gamma}=k_{B} T[x \ln x+(1-x) \ln (1-x)]
$$


i.e. to the configuration entropy of a perfectly disordered solid solution with no short-range order. Note that the excess term (eq. 3) may include a non-ideal configurational part, but it is not identified as such.

Finally, the Gibbs free energy of mixing is:

$$
G_{m i x}^{\gamma}=G^{\gamma}-(1-x) G_{F e}^{\prime \gamma}-x G_{N i}^{\prime \gamma}
$$

where $G_{F e}^{\prime \gamma}=G_{F e}^{\gamma}+G_{m a g}^{\gamma}(x=0)$ and $G_{N i}^{\prime \gamma}=G_{N i}^{\gamma}+G_{m a g}^{\gamma}(x=1)$ are the total Gibbs free energies of the pure metals, including the magnetic part.

\section{2. $\quad$ Ordered Phases}

In the study by Cacciamani et al. [18], the ordered phases $\mathrm{FeNi}_{3}\left(\mathrm{~L}_{2}\right), \mathrm{Fe}_{3} \mathrm{Ni}\left(\mathrm{L}_{2}\right)$ and $\mathrm{FeNi}\left(\mathrm{L}_{1}\right)$ are modeled within the framework of the Compound energy formalism (CEF) with 4 sublattices. An additional term is added to the Gibbs free energy of the $\gamma$ phase, which depends on the distribution of species on the different sublattices and on interaction energies (limited to the first nearest neighbors) fitted to ab initio calculations of the formation enthalpy of the perfectly ordered phase.

In the present work, we will use more detailed ab initio studies, involving both ordered and disordered configurations and summarized in the following section.

\section{B. Ab initio calculations}

\section{Density functional theory method}

In this work, the $0 \mathrm{~K}$ formation enthalpies of $\mathrm{Fe}-\mathrm{Ni}$ ordered and disodered phases are fitted to those computed in the ab initio study of Ref. [17]. The essential computational points are presented in the following.

The ab initio calculations were performed using density functional theory (DFT) with the projector augmented wave method $[35,36]$ as implemented in the VASP (Vienna Ab-initio Simulation Package) [37-39]. The generalized gradient approximation for the exchangecorrelation functional in the Perdew-Burke-Ernzerhof parametrization [40] was employed. All the calculations are spin-polarized. $3 d$ and $4 s$ electrons were considered as valence electrons. The plane-wave basis cutoff was set to $400 \mathrm{eV}$. The Methfessel-Paxton broadening 
scheme with a smearing width of $0.1 \mathrm{eV}$ was used [41]. The $k$-point grids were generated according to the cell size to achieve a $k$-sampling equivalent to a cubic unit cell with a $16 \times 16 \times 16$ shifted grid following the Monkhorst-Pack scheme [42]. The zero-point energies, which can be significant for light elements, have been calculated in the ordered phases. Their contribution to the mixing enthalpies is very small (typically 0.001 to $0.003 \mathrm{eV}$ ) and has been neglected in the following. FCC solid solutions were represented by Special Quasirandom Structures (SQS) [43] minimizing Warren-Cowley short-range order parameters [44, 45], with 128-atom and 108-atom supercells for anti-ferromagnetic-double-layer and ferromagnetic phases, respectively.

\section{2. $\quad$ Ordered Phases}

TABLE I. Formation enthalpies of FCC ordered phases in Fe-Ni (DFT calculations from [17]).

\begin{tabular}{|c|c|}
\hline Ordered phase & $H_{\text {for }}(\mathrm{eV} /$ atom $)$ \\
\hline $\mathrm{Fe}_{7} \mathrm{Ni}-\mathrm{cI} 32$ & 0.039273 \\
\hline $\mathrm{Fe}_{3} \mathrm{Ni}-\mathrm{L} 1_{2}$ & -0.01636 \\
\hline $\mathrm{Fe}_{3} \mathrm{Ni}-\mathrm{Z} 1$ & -0.04414 \\
\hline $\mathrm{Fe}_{2} \mathrm{Ni}-\mathrm{C} 11_{f}$ & -0.06991 \\
\hline $\mathrm{FeNi}-\mathrm{L} 1_{1}$ & -0.04040 \\
\hline FeNi-L1 $1_{0}$ & -0.10797 \\
\hline $\mathrm{Fe}_{2} \mathrm{Ni}-\mathrm{C} 11_{f}$ & -0.08064 \\
\hline $\mathrm{FeNi}_{2}-\mathrm{L}_{2}$ & -0.10879 \\
\hline $\mathrm{FeNi}_{7}-\mathrm{cI} 32$ & -0.04541 \\
\hline
\end{tabular}

The formation enthalpies of nine ordered structures on the FCC lattice, calculated by Kangming et al. [17] using the DFT method presented in the previous section, are given in Table I and Fig. 2. The ordered structures are ferromagnetic, except for $\mathrm{Fe}_{7} \mathrm{Ni}$-cI32 which is ferrimagnetic. The formation enthalpies are defined with the anti-ferromagnetic FCC iron and the ferromagnetic FCC nickel as reference states. The trends are the same as in a previous study by Mishin et al. [16]: only the FeNi-L1 $1_{0}$ and $\mathrm{FeNi}_{3}-\mathrm{L}_{2}$ phases are located on the convex hull (Fig. 2) and must therefore be stable at low temperature on the FCC 


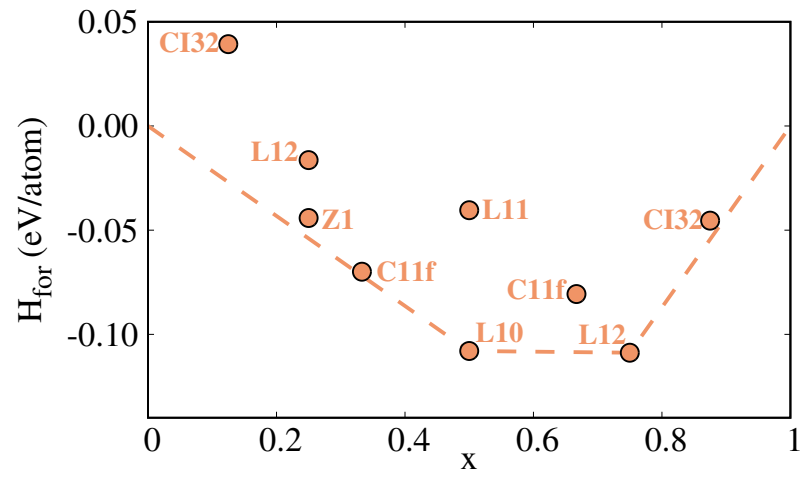

FIG. 2. Formations enthalpies of ordered FCC structures at $0 \mathrm{~K}$ (DFT calculations from [17]).

lattice. However the $\mathrm{FeNi}_{7}$-cI32 and $\mathrm{Fe}_{2} \mathrm{Ni}-\mathrm{C} 11_{f}$ phases are close to the stability limit.

\section{Special Quasi-Random Structures}

The formation enthalpies $H_{\text {for }}$ of special quasi-random structures (SQS) of different compositions have also been calculated in the same study with different magnetic states. These structures are representative of random solid solutions. They were generated by standard methods, with a minimization of Warren-Cowley short-range order parameters. They are described in Ref. [17], with a detailed analysis of their volume and magnetic moments. We just recall here the energetic results used for the PIM parametrization.

The most stable SQS are found to be double-layered anti-ferromagnetic for $x<0.184$ and ferromagnetic for $x>0.184$ (red circles in Fig. 3). One observes an asymmetrical evolution of $H_{f o r}$ with the composition, as already predicted in the study by Cacciamani et al., with mainly negative values (i.e. a tendency to order) and a minimum in the vicinity of the composition of the $\mathrm{FeNi}_{3}$ phase. However, the SQS values are significantly larger than the CALPHAD ones (Fig. 3), and are even slightly positive for $x<0.20$ (as already obtained by Sansa et al. [46], using a tight-binding approach).

\section{Pair interaction model}

We propose to reproduce the properties of Fe-Ni alloys with a model of concentrationand temperature-dependent pair interactions on a perfect FCC lattice. This pair interaction model (PIM) is based on a similar one developed for Monte Carlo simulations of BCC Fe-Cr 


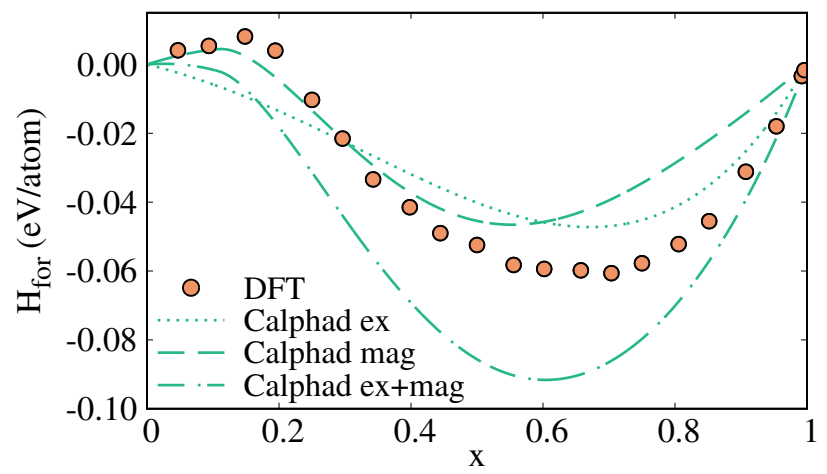

FIG. 3. Formations enthalpies at $0 \mathrm{~K}$ of quasi-random FCC structures (DFT calculations [17]) and FCC solid solutions (CALPHAD [18], with separate magnetic and excess contributions).

alloys [9, 47], which had however not been fitted systematically on a CALPHAD model, and did not explicitly distinguish a magnetic contribution.

The essential assumption of the PIM is that the Gibbs free energy (per atom) of a given configuration, i.e. a given distribution of $n_{F e}$ Fe atoms and $n_{N i}$ Ni atoms $\left(N=n_{F e}+n_{N i}\right)$ on the FCC lattice, can be written as a sum of interactions $g_{i j}^{(n)}(x, T)$ between pairs of $i$ and $j$ atoms on $n^{\text {th }}$ neighboring sites:

$$
G_{\text {conf }}=\frac{1}{N} \sum_{i j} g_{i j}^{(n)}(x, T)
$$

The pair interactions depend on the temperature, $T$, and the Ni concentration, $x$ (we will omit these dependencies in the following, to simplify the notations). The dependence on concentration is required to reproduce an asymmetric evolution of formation enthalpies, as observed in Fig. 2 and Fig. 3. The dependence on temperature describes the entropic contributions (electronic, vibrational and magnetic) other than the configuration entropy, so the $g_{i j}^{(n)}$ are indeed "pair Gibbs free energies" and can be written as: $g_{i j}^{(n)}=h_{i j}^{(n)}-T s_{i j}^{(n)}$ [48].

To facilitate the comparison with CALPHAD models, each interaction $g_{i j}^{(n)}$ is written as a sum of a non-magnetic $(\mathrm{nm})$ and a magnetic term $(\mathrm{mag}) . G_{\text {conf }}$ is therefore the sum of:

$$
G_{\text {conf }}^{n m}=\frac{1}{N} \sum_{i j} g_{i j}^{n m(n)}
$$

which accounts for the chemical and vibrational contributions, and of:

$$
G_{\text {conf }}^{m a g}=\frac{1}{N} \sum_{i j} g_{i j}^{\operatorname{mag}(n)}
$$


The total Gibbs free energy of the alloy is:

$$
G=G_{c o n f}-T S_{c o n f}
$$

where $S_{\text {conf }}$ is the entropy of configuration, which will be evaluated from Monte Carlo simulations.

The Gibbs free energy of mixing $G_{m i x}$ is:

$$
G_{m i x}=G-(1-x) G_{F e}^{\prime}-x G_{N i}^{\prime}
$$

$G_{F e}^{\prime}$ is the Gibbs free energy of pure iron, on the same FCC lattice:

$$
\begin{aligned}
G_{F e}^{\prime} & =G_{F e}^{n m}+G_{F e}^{m a g} \\
& =\sum_{n} \frac{z_{n}}{2}\left(g_{F e F e}^{n m(n)}+g_{F e F e}^{m a g(n)}\right) \\
& =\sum_{n} \frac{z_{n}}{2} g_{F e F e}^{(n)}
\end{aligned}
$$

where $z_{n}$ is the coordination number for the nearest neighbors $n$. The same expressions apply to pure nickel.

\section{High temperatures: fitting of the pair interactions to CALPHAD}

In the PIM, the Gibbs free energy of mixing of a perfectly disordered solid solution is:

$$
G_{m i x}(x, T)=x(1-x) \sum_{n} v_{n}(x, T)-T S_{i d}
$$

where the ordering parameters $v_{n}$ are defined as:

$$
v_{n}(x, T)=g_{F e N i}^{(n)}-\frac{1}{2}\left(g_{F e F e}^{(n)}+g_{N i N i}^{(n)}\right)
$$

To reproduce the properties of the CALPHAD model, we identify the non-magnetic part of the ordering parameters (in eq. 15) to the excess Gibbs free energy of CALPHAD (eq. 3):

$$
\sum_{n} v_{n}^{n m}(x, T)=\frac{G_{e x}^{\gamma}}{x(1-x)}
$$

and their magnetic part to the magnetic Gibbs free energy of CALPHAD (eq. 4):

$$
\sum_{n} v_{n}^{m a g}(x, T)=\frac{G_{m a g}^{\gamma}}{x(1-x)}
$$


The fitting of the PIM parameters on CALPHAD can be summarized to the equations 16-18. It is worth to notice that it is based on an approximation: eq. 15 is exact only for a disordered solid solution, i.e. at sufficiently high temperatures. In the real solid solution, a short-range order may exist, and the configuration entropy does not reduced to an ideal term.

\section{Low temperatures: fitting of the pair interactions to ab initio calculations}

To reproduce the properties of a solid solution at $0 \mathrm{~K}$, the same method can be used by fitting $v_{n}(x, T)$ on the formation enthalpies of SQS calculated by DFT. The magnetic part remains fitted to the magnetic model of CALPHAD (eq. 18) and the non-magnetic part is fitted so that the sum of the magnetic and non-magnetic contributions of the PIM reproduces the DFT formation enthalpies. A good agreement is obtained with a RedlichKister polynomial of order 5 (instead of 2 for $G_{e x}^{\gamma}$ in ref. [18]), as shown in Fig. 4 (with respectively the magnetic part, the non-magnetic part and the total mixing enthalpies of the PIM). The fitting coefficients $L_{j}^{D F T}$ of the polynomial are given in Table II, corresponding to the orange curve in Fig. 4. As mentioned above, this gives mixing enthalpies above those predicted by CALPHAD at $0 \mathrm{~K}$.

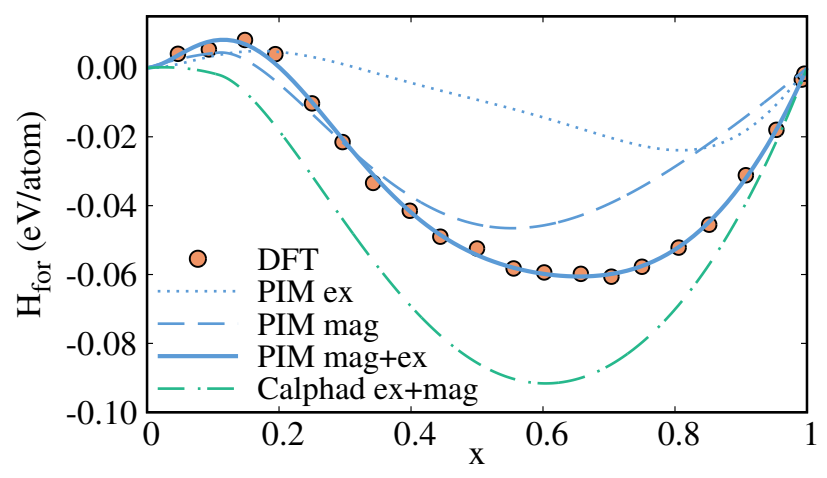

FIG. 4. Formations enthalpies of quasi-random FCC Structures at $0 \mathrm{~K}$ : ab initio calculations (DFT) and pair interaction model (PIM, with the excess and magnetic contributions). The formations enthalpy of the $\gamma$ solid solution of CALPHAD is also shown for comparison.

This fit of pair interactions (using eq. 15) to the CALPHAD model or to the formation enthalpies of SQS, only involves the sum of $v_{n}$ and can be done with any range $n$ of interac- 
TABLE II. The coefficients $L_{j}^{D F T}$ of the Redlich-Kister polynomial for the excess enthalpy of mixing of the $\gamma$ solid solution of the PIM (in eV).

\begin{tabular}{|c|c|c|c|}
\hline$L_{0}^{D F T}$ & $L_{1}^{D F T}$ & $L^{D F T}$ & \\
\hline
\end{tabular}

$-0.036960 .09631-0.047220 .21141-0.04752-0.18389$

tions. However, it is well known that in FCC ordered structures, the phase diagram and in particular the order-disorder temperatures, depend strongly on the ratio $=v_{2} / v_{1}$ [49]. The fit is also independent of the choice of the composition $x$ (local or global) in eq. 18 and 17 . Therefore, it does not provide a very accurate description of the interatomic interactions in Fe-Ni alloys.

To get a better description of the thermodynamic properties, the range of interactions and the ratio between the interactions at different distances is fitted to the DFT calculations of the formation enthalpies of the ordered phases (Table I).

With only first nearest neighbors (nn) interactions (Fig. 5(a)), the PIM model underestimates the formation enthalpies of the ordered phases, especially those of the FeNi- $\mathrm{L} 1_{0}$ and $\mathrm{FeNi}_{3}-\mathrm{L}_{2}$ phases.

A better result (Fig. 5(b)) is obtained with first and second nn pair interactions, taking a constant ratio $\alpha=v_{2} / v_{1}=-0.7$ (the agreement is very sensitive to the value of $\alpha$, except in the range $\alpha \in[-0.6,-0.7])$. The formation enthalpies obtained with first and second $\mathrm{nn}$ interactions are close to those of the DFT calculations, in particular for the two stable phases $\mathrm{FeNi}-\mathrm{L}_{0}$ and $\mathrm{FeNi}_{3}-\mathrm{L}_{2}$ (Fig. 5). The least well reproduced is that of the iron-rich cI32 phase, which is unstable.

Note that in this fitting procedure, the local composition around a Fe-Ni pair has been defined as the average $\mathrm{Ni}$ atomic fraction around the first and second nearest neighbors of the pair, excluding the two atoms which compose it. An atom neighboring the two atoms of the pair is counted twice (so that with 12 first and 6 nearest neighbors, a pair is surrounded by 0 to $34 \mathrm{Ni}$ atoms). Using this definition, the formation enthalpies of the different ordered phases are the functions of $v_{2}$ and $v_{1}$ given in Table III, together with the values of the local composition $x_{1}$ and $x_{2}$ around the first and second nn Fe-Ni pairs. Other definitions of the local composition are possible and we have tested some of them (taking into account the two atoms of the pairs, or counting each surrounding $\mathrm{Ni}$ atom only once). The definition 
chosen here gives a slightly better fit, although the differences are small.

We did not obtain significantly better results by introducing third and fourth nn interactions. In the following we will therefore restrict to the PIM with first and second nn interactions and $\alpha=-0.7$, corresponding to the results shown in Fig. 4 and $5(\mathrm{~b})$.



(a)

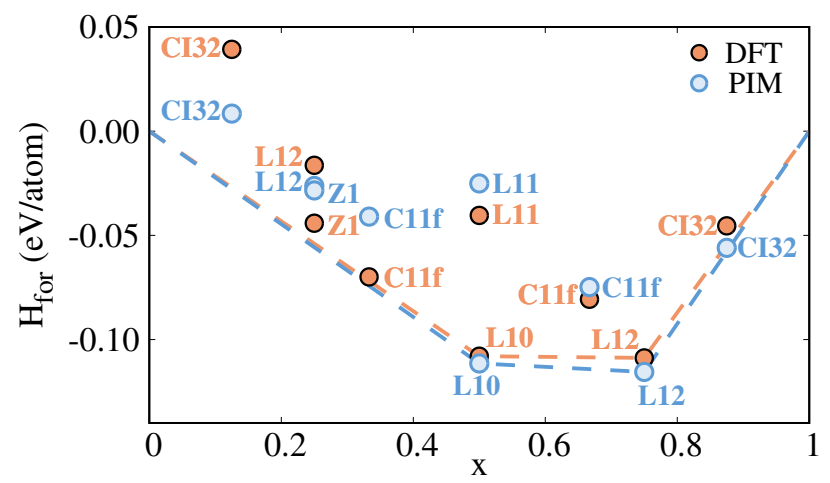

(b)

FIG. 5. Formations enthalpies of FCC ordered phases at $0 \mathrm{~K}$ : ab initio calculations (DFT, full circles) and pair interaction model (PIM, open circles) with (a) only first nn interactions, (b) first and second nn interactions, $\alpha=v_{2} / v_{1}=-0.7$

\section{Transition between parameters at high and low temperatures}

The final PIM will therefore use first and second neighbor pair interactions with a constant $\alpha=v_{2} / v_{1}=-0.7$ ratio:

- At $0 \mathrm{~K}$ the variations of $v_{2}$ and $v_{1}$ with the composition are fitted to the formation enthalpies of SQS and ordered structures calculated by Li and Fu [17] using DFT methods, 
TABLE III. Formation enthalpies of FCC ordered phases in a pair interaction model with first and secong nearest neighbor interactions.

\begin{tabular}{lcll}
\hline Ordered phases & $x_{1}$ & $x_{2}$ & $H_{\text {for }}^{P I M}$ \\
\hline $\mathrm{Fe}_{7} \mathrm{Ni}-\mathrm{cI} 32$ & $\frac{1}{34}$ & $\frac{5}{34}$ & $\frac{3}{2} v_{1}+\frac{3}{4} v_{2}$ \\
$\mathrm{Fe}_{3} \mathrm{Ni}-\mathrm{L} 1_{2}$ & $\frac{9}{34}$ & 0 & $3 v_{1}$ \\
$\mathrm{Fe}_{3} \mathrm{Ni}-\mathrm{Z} 1$ & $\frac{11}{34}$ & $\frac{9}{34}$ & $2 v_{1}+\frac{1}{2} v_{2}$ \\
$\mathrm{Fe}_{2} \mathrm{Ni}-\mathrm{C} 11_{f}$ & $\frac{6}{17}$ & $\frac{6}{17}$ & $\frac{8}{3} v_{1}+\frac{2}{3} v_{2}$ \\
$\mathrm{FeNi}_{-}-\mathrm{L} 1_{1}$ & $\frac{1}{2}$ & $\frac{1}{2}$ & $3 v_{1}+3 v_{2}$ \\
$\mathrm{FeNi}_{-} 1_{0}$ & $\frac{1}{2}$ & 0 & $4 v_{1}$ \\
$\mathrm{FeNi}_{2}-\mathrm{C} 11_{f}$ & $\frac{11}{17}$ & $\frac{11}{17}$ & $\frac{8}{3} v_{1}+\frac{2}{3} v_{2}$ \\
$\mathrm{FeNi}_{3}-\mathrm{L} 1_{2}$ & $\frac{25}{34}$ & 1 & $3 v_{1}$ \\
$\mathrm{FeNi}_{7}-\mathrm{cI} 32$ & $\frac{33}{34}$ & $\frac{29}{34}$ & $\frac{3}{2} v_{1}+\frac{3}{4} v_{2}$ \\
\hline
\end{tabular}

as summarized in fig. 5 and 4 ;

- At high temperatures the variations of $v_{2}$ and $v_{1}$ with the composition are fitted to the Gibbs free energies of the $\gamma$ solid solution, from the CALPHAD study of Cacciamani et al. [18];

These two sets of parameters differ only in the non-magnetic part of parameters $v_{2}$ and $v_{1}$, which is described by Redlich-Kister polynomials having different order and coefficients (respectively $L_{i}^{D F T}$ and $L_{i}^{C A L P H A D}$ ). The final parameters are obtained by using coefficients $L_{i}^{\text {PIM }}(T)$ which evolves gradually from one to the other according to:

$$
\begin{aligned}
L_{j}^{P I M}(T) & =\exp \left(-\frac{T}{T_{j}}\right) L_{j}^{D F T} \\
& +\left[1-\exp \left(-\frac{T}{T_{j}}\right)\right] L_{j}^{C A L P H A D}(T)
\end{aligned}
$$

with $T_{j}=400 \mathrm{~K}$ for $j=0,1,2$ and $T_{j}=80 \mathrm{~K}$ for $j=3,4,5$. The example of coefficient $L_{2}^{P I M}$ is given in fig. 6 . The temperatures $T_{j}$ have been chosen so as to give the Gibbs free energy of CALPHAD for $T>1000 \mathrm{~K}$, at temperatures where it is derived from numerous and reliable experimental data. The influence of these transition temperatures on the phase diagram will be discussed later.

Let us note finally that the Gibbs free energy of mixing of the solid solution, the formation enthalpies of of SQS or ordered structures and the FCC phase diagram, depend only on the 
parameters $v_{n}$. We have chosen to take $g_{F e F e}^{(n)}$ and $g_{N i N i}^{(n)}$ interactions independent of the concentration, but dependent on the temperature and adjusted to the free enthalpies of the pure metals (eq. 12). Only the $g_{F e N i}^{(n)}$ interactions are dependent on local concentration. This choice does not affect the results of the present study, but it allows a better description of the properties of point and diffusion defects [9], which we will address in future work. It also makes the Monte Carlo simulations slightly less time consuming.



FIG. 6. The evolution of the coefficient $L_{2}^{P I M}(T)$ with the temperature.

\section{MONTE CARLO SIMULATIONS}

We will now use Monte Carlo simulations to measure of Gibbs free energies of the PIM (including the configuration entropy) at different compositions and temperatures and build the FCC phase diagram.

\section{A. Semi-grand canonical isotherms}

The equilibrium properties of the PIM are determined by Monte Carlo simulations carried out in the semi-grand canonical ensemble. In general, we use a system of $N=4 \times 16^{3}$ atoms, with periodic boundary conditions. Exchanges are tried between a randomly chosen atom of the system and an atom taken in a reservoir, with a given difference of chemical potential $\Delta \mu=\mu_{N i}-\mu_{F e}$. By changing $\Delta \mu$, one modifies the equilibrium concentration (Fig. 8 and 9). A total of 500 increments for a interval of $1 \mathrm{eV}$ in $\Delta \mu$ are used to go from pure iron to pure nickel, and then 500 increments to go the other way. For each value of $\Delta \mu, 5 \times 10^{6}$ attempts of atomic exchange (or Monte Carlo steps, MCS) are performed 
before measuring the equilibrium composition and order parameters. For building the phase diagram of Fig. 16, a larger system of $N=4 \times 24^{3}$ atoms and much smaller increments of $\Delta \mu$ are used, in order to get a better precision (up to 100 increments for a interval of 0.04 in $\Delta \mu)$.

To identify the different ordered phases, the FCC lattice is divided into 4 simple cubic sublattices, shifted by a distance $a / 2$ in the $x, y, z$ directions [50]. We measure the $\mathrm{Ni}$ concentration on each sublattice, and the long range order parameter defined as:

$$
\eta=\frac{1}{4} \sum_{i=1}^{4}\left|\frac{x_{i}}{x}-1\right|
$$

where $x_{i}$ is the $\mathrm{Ni}$ concentration on the sublattice $i$. With this definition, $\eta=1$ in the perfect FeNi-L1 $1_{0}$ structure and $\eta=0.5$ in the perfect $\mathrm{FeNi}_{3}-\mathrm{L}_{2}$ structure.

The short range order is characterized by the Warren-Cowley parameters for the first and second nearest neighbors:

$$
\sigma_{i}=1-\frac{f_{N i}^{(i)}}{x}
$$

where $f_{N i}^{(i)}$ is the average fraction of $\mathrm{Ni}$ atoms among the $i^{\text {th }} \mathrm{nn}$ of the Fe atoms. For a perfect $\mathrm{L}_{2}$ ordered phase, $\sigma_{1}=-0.33$ and $\sigma_{2}=+1$.

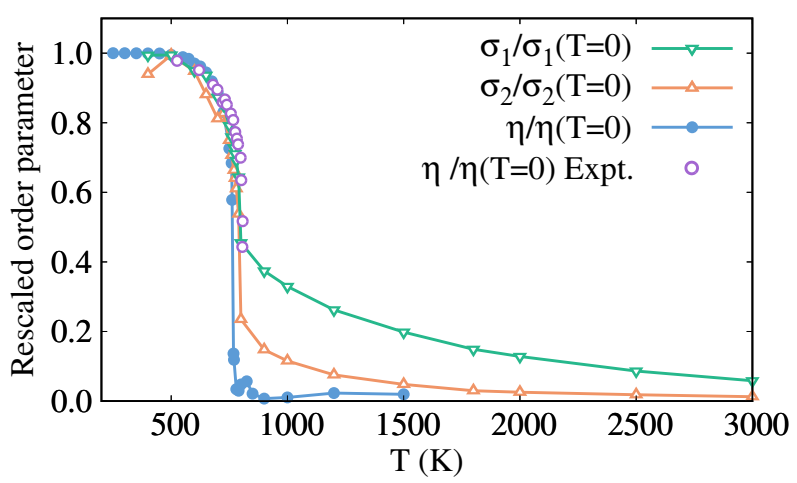

FIG. 7. Evolution the long-range parameter $\eta$ and of the short-range order parameters $\sigma_{1}$ and $\sigma_{2}$ in $\mathrm{FeNi}_{3}$ as a function of $T$ (Monte Carlo simulations and experiments by Kozlov et al. [51]).

The evolution of the long-range $\eta$ (eq. 20), and of the short range order parameters $\sigma_{1}$ and $\sigma_{2}$ (eq. 21), in an alloy of composition $\mathrm{FeNi}_{3}$ as a function of the temperature, is shown in Fig. 7. The $\mathrm{L}_{2}$ ordered phase is found to be stable up to $765 \mathrm{~K}$ (instead of $790 \mathrm{~K}$ for CALPHAD [18]). The evolution of $\eta$ is in good agreement with the experiments of Kozlov et al. [51] (which gives a slightly higher ordering temperature: $807 \mathrm{~K}$ ). The discontinuity at 
the order/disorder temperature indicates a first-order transition. A significant short range order remains well above the disordering temperature, especially between the first nearest neighbors.

Two examples of isotherms $x=f(\Delta \mu)$, at $T=1000 \mathrm{~K}$ and $T=600 \mathrm{~K}$ are shown in Fig. 8 and 9. At $1000 \mathrm{~K}, x(\Delta \mu)$ evolves continuously, with $\eta \simeq 0$ : the disordered solid solution is stable in the whole composition range. At $600 \mathrm{~K}, \eta \simeq 0.76$ to 0.53 between $\Delta \mu=-0.188$ and $-0.068 \mathrm{eV}$, which corresponds to an over stoichiometric $\mathrm{L}_{0}$ phase; and $\eta \simeq 0.49$ between $\Delta \mu=-0.05$ and $+0.3 \mathrm{eV}$, which corresponds to an almost stoichiometric $\mathrm{L}_{2}$ phase. Discontinuities and hysteresis on the $x(\Delta \mu)$ curve indicate first order transitions and the limits of two-phase domains.

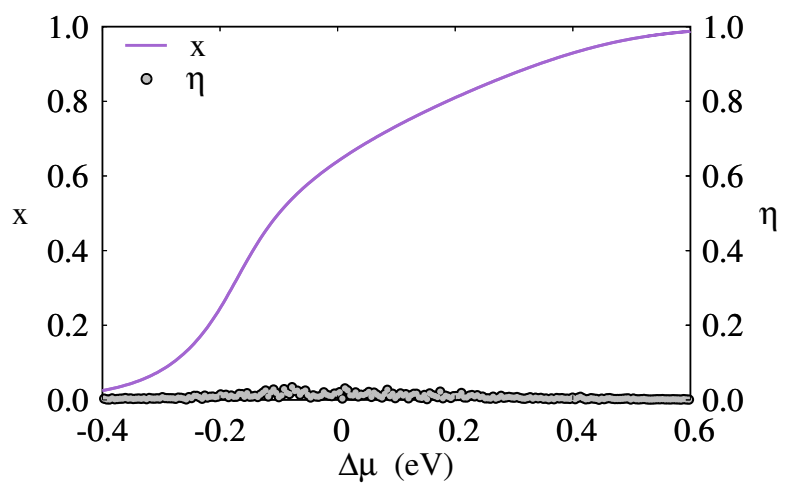

FIG. 8. Monte Carlo simulations: evolution of the Ni concentration $x$ and long-range order parameter $\eta$ of the PIM for Fe-Ni alloys at $1000 \mathrm{~K}$, as a function of the difference in chemical potentials $\Delta \mu=\mu_{N i}-\mu_{F e}$.

\section{B. Gibbs free energy of mixing}

Using the definition of chemical potentials: $\mu_{i}=\left(\partial G / \partial n_{i}\right)_{T, P, n_{j}}$ and integrating the $\Delta \mu(x)$ curve, we obtain the Gibbs free energy of mixing $G_{m i x}$. The results obtained at different temperatures can be directly compared with the $G_{m i x}$ of the CALPHAD study [18]. In each case, one can also compare separately, the enthalpy $H_{m i x}$ and entropy $S_{m i x}$ of mixing, as well as the magnetic, excess and configuration contributions.

Fig. 10 for example, gives the Gibbs free energy of mixing of the Fe-Ni solid solution at $T=1500 \mathrm{~K}$. The PIM is in very good agreement with the CALPHAD study. At this high temperature (well above $T_{c}$ ), the magnetic contribution is negligible. However it is 




FIG. 9. Monte Carlo simulations: evolution of the Ni concentration $x$ and long-range order parameter $\eta$ of the PIM for Fe-Ni alloys at $600 \mathrm{~K}$, as a function of the difference in chemical potentials $\Delta \mu=\mu_{N i}-\mu_{F e}$.

worth to notice that the excess contribution is slightly lower in the PIM, with a minimum of $-0.038 \mathrm{eV}$ at $x=0.69$ instead of $-0.033 \mathrm{eV}$ in CALPHAD. The difference is due to the fact that in the PIM, some short range order remains in the $\gamma$ solid solution, even at this high temperature (Fig. 7).



FIG. 10. Gibbs free energy of mixing of the Fe-Ni solid solutions at 1500 K: PIM (dotted lines) vs CALPHAD (full lines), with the separate magnetic, excess and configurational entropic contributions.

The effect is clearer if one separates the enthalpic and entropic contributions of $G_{m i x}=$ $H_{\text {mix }}-T S_{\text {mix }}$. The enthalpy of mixing (Fig. 11) is dominated by the excess term. Due to the remaining short range order $\left(\sigma_{1}=-0.066\right.$ and $\left.\sigma_{2}=0.048\right)$, the PIM gives a minimum of $H_{m i x}=-0.054 \mathrm{eV}$ at $x=0.67$ instead of $-0.047 \mathrm{eV}$ in CALPHAD. This discrepancy on $H_{m i x}$ only disappear at very high temperature. At $3000 \mathrm{~K}$ (i.e. above the liquidus), 
the maximum difference between the PIM and CALPHAD is still $\Delta H_{\text {mix }}=0.0022 \mathrm{eV}$ (for $\sigma_{1}=0.06$ and $\left.\sigma_{2}=0.013\right)$. It becomes negligible only above $5000 \mathrm{~K}$.

The entropy of mixing of the the PIM (Fig. 12) is dominated by the entropy of configuration, which is very close to the ideal $S_{i d}$ of CALPHAD. In the PIM as in CALPHAD, the excess and magnetic contributions to the entropy of mixing are less important, and negative (except below $x \simeq 0.1$ ). At $x=0.67$, the discrepancy due to the short range order is only $S_{\text {conf }}-S_{i d}=0.15 \times 10^{-5} \mathrm{eV} /($ atom $\cdot \mathrm{K})$, which corresponds to a difference of $T\left(S_{\text {conf }}-S_{i d}\right)=-0.00225 \mathrm{eV} /$ atom. The difference on $S_{m i x}$ partly compensate the one on $H_{m i x}$, which explain the good agreement on $G_{m i x}$ between the PIM and CALPHAD, even below $1500 \mathrm{~K}$, when the short range order increases. The $G_{m i x}$ of the PIM and CALPHAD are therefore in very good agreement in the whole range of composition and temperature where the $\gamma$ solid solution is stable (Fig. 13).

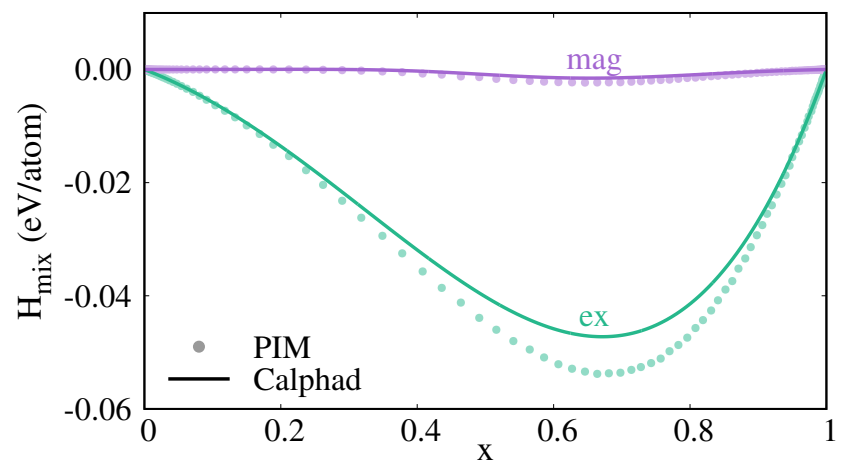

FIG. 11. Enthaply of mixing of the Fe-Ni solid solutions at $1500 \mathrm{~K}$ : PIM (dotted lines) vs CALPHAD (full lines), with separate magnetic and excess contributions.

The difference between the Gibbs free energy of mixing of the PIM and CALPHAD [Fig. 14(a)] slightly increases at lower temperatures, when the ordered phase $\mathrm{L}_{2}$ stabilizes, i.e. when the long-range order parameter $\eta$ is close to 0.5 , between $x \simeq 0.6$ and $x \simeq 0.82$ [Fig. 14(b)]. This is not surprising since the PIM parameters at low temperatures are not fitted on CALPHAD, but on DFT calculations which give a different energetic landscape, especially for the disordered phase (section IIC). In spite of this difference, the $G_{m i x}(x)$ curve of the PIM is still in good agreement with CALPHAD at $T=700 \mathrm{~K}$.

At $650 \mathrm{~K}$, the agreement between the Gibbs free energy of mixing of the PIM and CALPHAD is still quite good for the compositions where the $\gamma$ solid solution and the $\mathrm{L}_{2}$ phase 


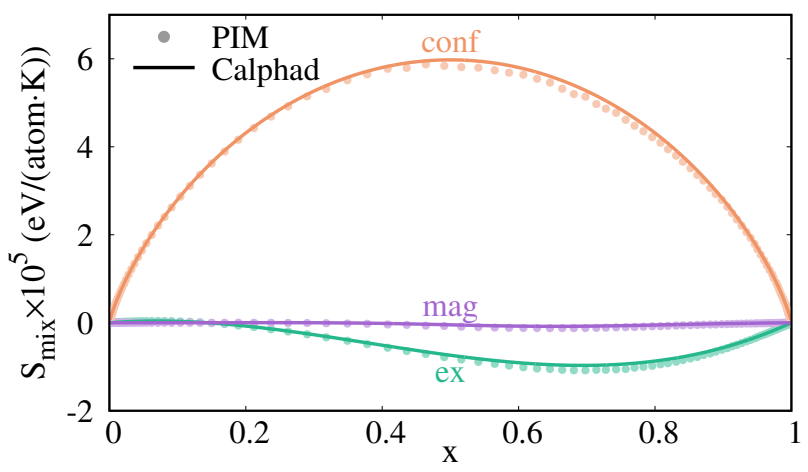

FIG. 12. Entropy of mixing of the Fe-Ni solid solutions at $1500 \mathrm{~K}$ : PIM (dotted lines) vs CALPHAD (full lines), with separate magnetic, excess and configurational contributions.

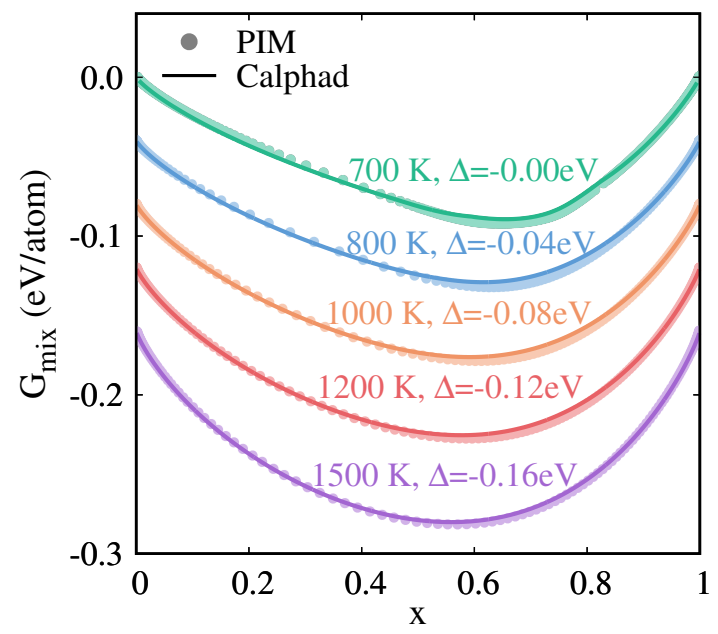

FIG. 13. Gibbs free energy of mixing of the Fe-Ni solid solution of the PIM and CALPHAD [18], at different temperatures. For the sake of clarity, each curve is shifted downwards by a constant $\Delta$ given on the figure.

are stable [Fig. 15(a)]. However the evolution of the long-range parameter $\eta$ as a function of the nickel concentration $x$ now displays two bumps [Fig. 15(b)]. The second one (between $x \simeq 0.63$ and $x \simeq 0.85)$ still corresponds to the $\mathrm{L}_{2}$ phase. The phase is almost perfectly ordered for the stoichiometric composition $\mathrm{FeNi}_{3}(\eta \simeq 0.5$ for $\left.x=0.75)\right)$. However, the PIM predicts that the FeNi-L1 $1_{0}$ phase is stable between $x \simeq 0.52$ and 0.63 , while it only appears at lower temperature (below $316 \mathrm{~K}$ ) according to the CALPHAD model [18]. Note that it is not perfectly ordered ( $\eta \simeq 0.6$ instead of 1 for the perfect order), because it is slightly 


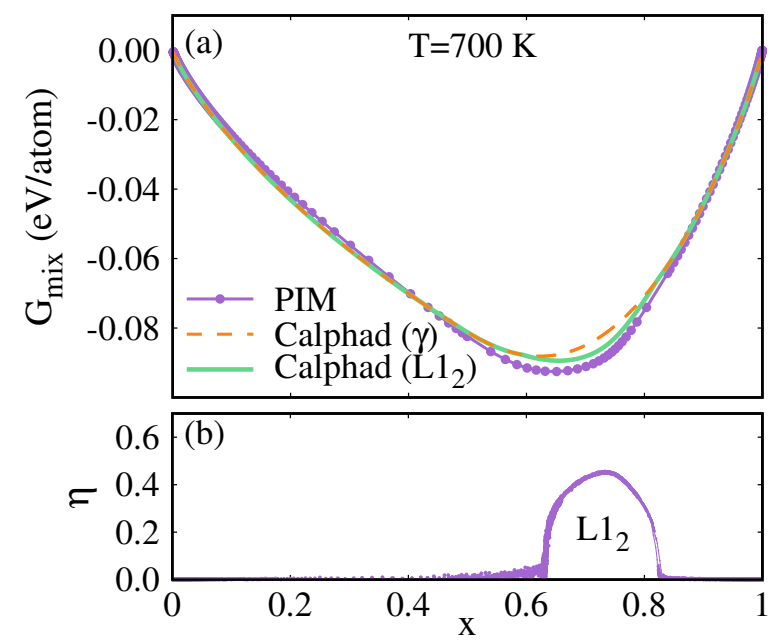

FIG. 14. (a) Gibbs free energy of mixing of Calphad and the PIM and (b) long range order parameter of the PIM, for Fe-Ni alloys at $700 \mathrm{~K}$.

non-stoichiometric $(x>0.5)$ and because $650 \mathrm{~K}$ is close to its order disorder-temperature $(680 \mathrm{~K})$.
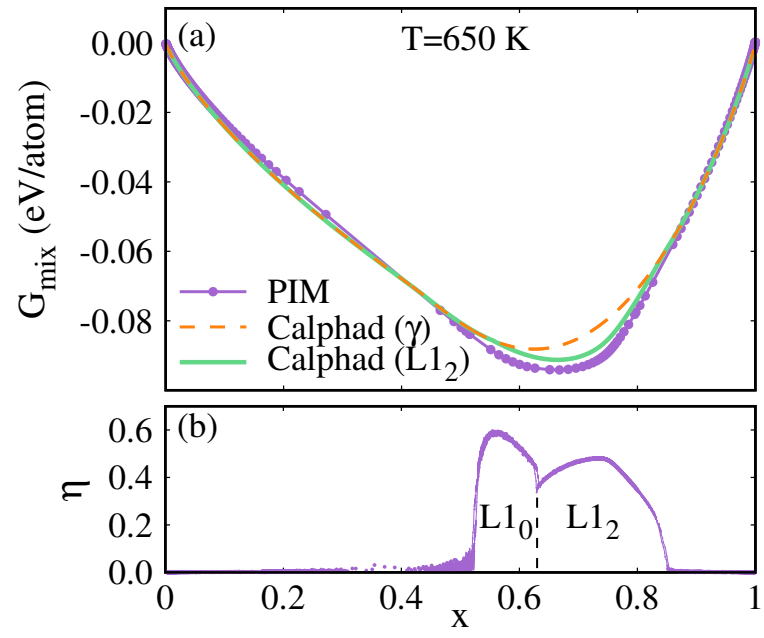

FIG. 15. (a) Gibbs free energy of mixing of Calphad and the PIM and (b) long range order parameter of the PIM, for Fe-Ni alloys at $650 \mathrm{~K}$.

\section{FCC phase diagram}

The FCC phase diagram of the Fe-Ni system predicted by the PIM is shown in Fig. 16 and compared with experimental data [52-55]. It can be also compared to the FCC diagram 
of Cacciamani et al. (Fig. 8 in [18]).

The ordering temperatures of the $\mathrm{FeNi}_{3}-\mathrm{L}_{2}$ phase are slightly different: the PIM gives $765 \mathrm{~K}$, a little lower than $790 \mathrm{~K}$ for the CALPHAD study of Cacciamani et al. [18] (which is adjusted to the experimental value). As in CALPHAD, the limits of the two-phase domain $\mathrm{FeNi}_{3}+\gamma$ for $x>0.75$, are slightly shifted towards lower values $(\Delta x \simeq-0.04$ at $571 \mathrm{~K})$ by comparison with the experiments by Heumann et al. [53]. And as in CALPHAD, the width of the two-phase domain $\gamma+\mathrm{FeNi}_{3}$ at $x<0.75$ is smaller than the one of the two-phase domain at $x>0.75$ (earlier CALPHAD studies predicted a larger two-phase field, as in Fig. 1). Here it should be noted that, except in the vicinity of the ordering temperature at $x=0.75$, the experimental data for these two-phase fields are going back to 1963 [53] and are only available for one temperature.

The discrepancy is more important for the FeNi- $1_{0}$ phase: the PIM predicts an ordering temperature of $680 \mathrm{~K}$ instead of $316 \mathrm{~K}$ for CALPHAD. The CVM study of Mohri et al. [26] predicts an intermediate ordering temperature $(483 \mathrm{~K})$. A direct estimation by DFT calculations (taking into account the vibrational entropy, but not the configurational entropy of the $\mathrm{FeNi}-\mathrm{L} 1_{0}$ phase) gives $640 \mathrm{~K}$. There is no precise experimental measurement available for the evolution of the degree of order as a function of the temperature (as for the $\mathrm{FeNi}_{3}$ phase, in Fig. 7), but the experimental observations under electron irradiation by Reuter et al. [23] suggest an ordering temperature of approx. $593 \mathrm{~K}$.

As in CALPHAD, the FCC phase diagram of the PIM also displays a two-phase field with an equilibrium between a ferromagnetic $\left(\gamma_{f}\right)$ and a paramagnetic $\left(\gamma_{p}\right)$ solid solution, at $x=0.4$ and below $T=660 \mathrm{~K}$. This phase separation has been first predicted by Chuang et al. [56], but it has not been confirmed experimentally. The PIM is able to reproduce this two-phase field because it includes the magnetic contribution of the CALPHAD model. It is however more limited in temperatures than in ref. [18], because of the higher stability of the FeNi-L1 0 phase in the PIM, which limits its extension below $680 \mathrm{~K}$.

The phase diagram of the PIM without the magnetic contribution is shown in Fig. 17. As in the study by Cacciamani et al., the non-magnetic phase diagram reduces the critical temperature of $L 1_{2}$ by approx. $118 \mathrm{~K}$ and shows no $\gamma_{f}-\gamma_{p}$ two-phase field.

Finally, let us recall that the parameters of the PIM and the results of section III have been obtained with parameters fitted to DFT calculations at $0 \mathrm{~K}$, the CALPHAD data of ref. [18] at high temperatures, and a transition between the high and low temperature regimes 


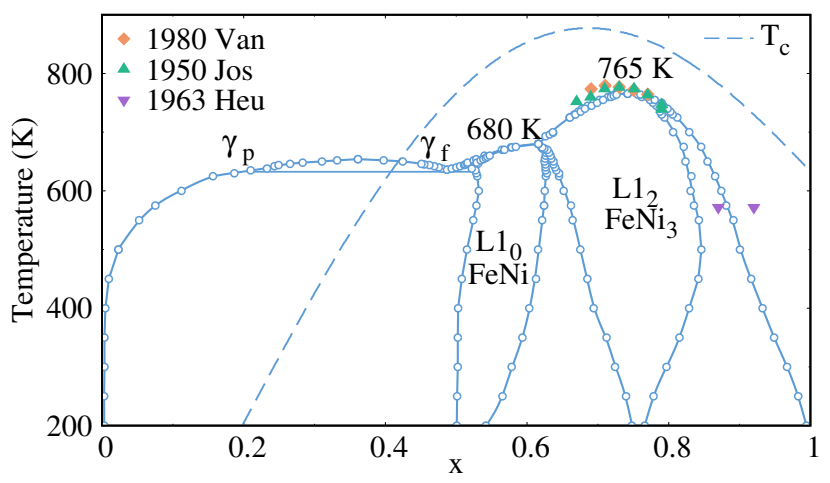

(a)

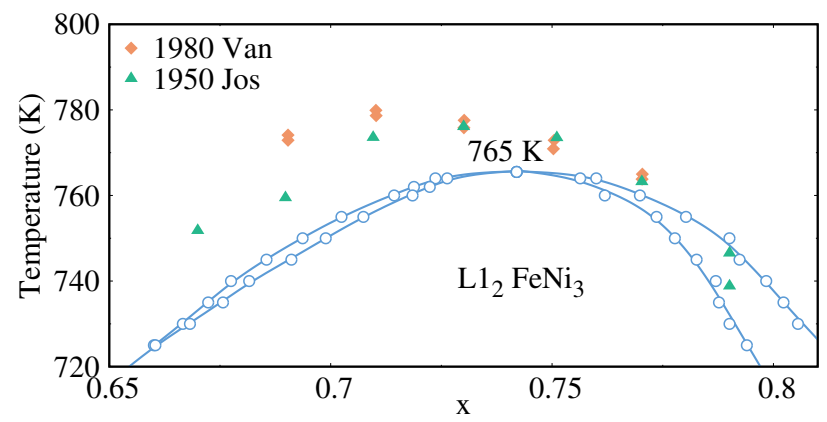

(b)

FIG. 16. (a) The Fe-Ni FCC phase diagram: comparison between the PIM and experiments (1950 Jos $=[52], 1963 \mathrm{Heu}=[53], 1980 \mathrm{Van}=[54,55] .$, (b) zoom in the region of the L1 2 ordering temperature.

controlled by the exponential interpolation of Eq. 17. With the chosen $T_{j}$ temperatures, the Redlich-Kister coefficients of the PIM are almost identical to those of CALPHAD above approximately $1000 \mathrm{~K}$. To assess the effect of this choice on the phase diagram, we have performed some simulations with a different set of paramaters: $T_{j}=50 \mathrm{~K}$ for $j=0,1,2$ and $T_{j}=10 \mathrm{~K}$ for $j=3,4,5$ (using the same notation as in II C 3 ). With these parameters, the Redlich-Kister coefficients of the PIM becomes almost identical to those of CALPHAD at a lower temperature (approx. $315 \mathrm{~K}$ ). The resulting phase diagram is shown in Fig. 18. It is not very different from the previous one (Fig. 16(a)), except from a moderate increase of the ordering temperatures of the $\mathrm{L}_{0}$ and $\mathrm{L} 1_{2}$ phases. The reason is that with the new parameters, the ordering tendency is a little more pronounced below $1000 \mathrm{~K}$ (Fig. 4). As a consequence, the $\gamma_{p}-\gamma_{f}$ two-phase domain almost completely disappears. 


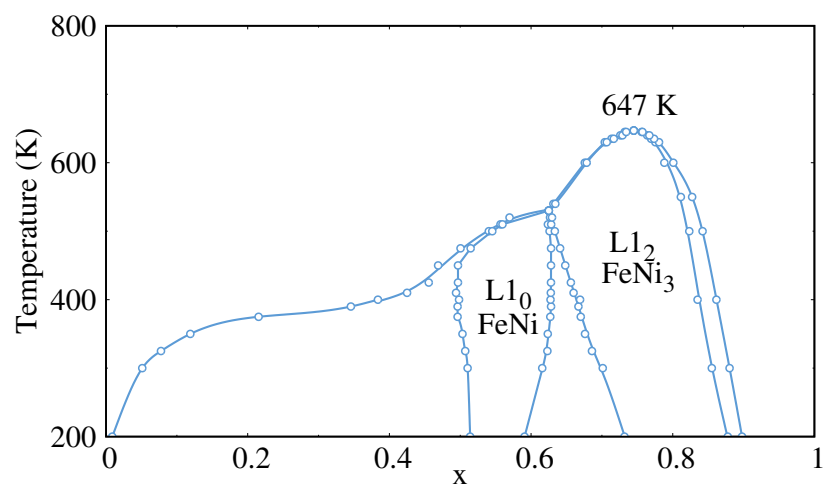

FIG. 17. The Fe-Ni FCC phase diagram of the PIM without magnetic contribution

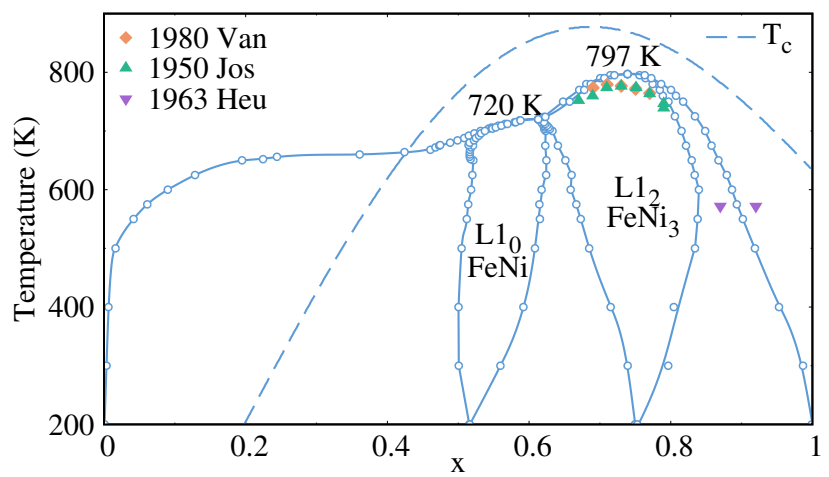

FIG. 18. The Fe-Ni FCC phase diagram of the PIM using a different transition between high and low temperature parameters.

\section{DISCUSSIONS AND CONCLUSIONS}

We have presented in this study a method for developing a pair interaction model for $\mathrm{Fe}-\mathrm{Ni}$ alloys, fitted at $0 \mathrm{~K}$ on the enthalpies of formation of ordered and disordered structures (computed by first-principle methods) and at high temperatures on the Gibbs free energy of the $\gamma$ solid solution (as given by a CALPHAD study and its underlying experimental data).

Thanks to the temperature and concentration dependence of the pair interactions, the PIM is able to reproduce precisely these two types of energetic properties, and to distinguish between excess, magnetic and configurational entropic contributions. The identification between CALPHAD and the PIM is not perfect, because the configurational entropy and short-range order in the solid solution is described more approximately in CALPHAD methods than in the Monte Carlo simulations used to determine the equilibrium properties 
of the PIM. The CALPHAD method is more flexible than an atomistic model: the properties of each phase can be adjusted very precisely and independently on the experiments. The PIM imposes some constraints but, combined with Monte Carlo simulations, it ensures a consistent description of the short- and long-range order and of the entropy of configuration.

Despite these differences, the Gibbs free enthalpies of mixing of the $\gamma$ solid solution, as given by CALPHAD, are very well reproduced by the PIM, throughout the composition and temperature range of stability of the phase. The Gibbs free enthalpy of the $\mathrm{FeNi}_{3}-\mathrm{L}_{2}$ phase is also well reproduce, although the parameters of the PIM and CALPHAD for that phase are not fitted on the same DFT calculations. At high temperatures, the FCC phase diagram involves only these two phases, and both methods give similar results (especially for the order-disorder transition in the vicinity of $\mathrm{FeNi}_{3}$, and for the $\gamma_{p}-\gamma_{f}$ phase separation).

On the other hand, both models predict that the FeNi-L1 $1_{0}$ phase is stable at low temperature, but with different ordering temperatures. Taking the electron irradiation experiments [23] as a reference, it seems that CALPHAD underestimates the ordering temperature, while the PIM overestimates it. It should be noted here that alloys under irradiation may be not fully at equilibrium, so that no real experimental thermodynamic data are available for this phase; and that both the CALPHAD model and the PIM are only fitted to DFT calculations of enthalpies of formation at $0 \mathrm{~K}$. The PIM and CALPHAD should therefore be both improved to properly describe this phase. One possibility is to use DFT methods to compute finite temperature contributions. These methods are computationally expansive, but can separate each energetic contribution and provide results at intermediate temperature (say, between 0 and $400^{\circ} \mathrm{C}$ ), where experimental results are rarer and perhaps less accurate. An example is given in the study of $\mathrm{K}$. Li and C.-C. Fu [17], which shows that the vibrational entropy decreases the ordering temperatures of the $\mathrm{L}_{0}$ and $\mathrm{L}_{2}$ phases by respectively 280 and $200 \mathrm{~K}$.

In spite of this limitation, the PIM model gives a satisfactory description of the $\gamma$ solid solution and of the $\mathrm{L}_{2}$ phase. Of course, such a model is necessarily dependent on the CALPHAD data it uses. For the magnetic contribution for example, we rely on the recent study by Cacciamani et al., which is itself based on a large experimental database (with measurements of specific heats, Curie temperatures, magnetic moments, etc. of $\gamma$ solid solutions with various compositions, described in ref. $[18,57])$. For the same reason, our model takes into account, but cannot distinguish between energetic contributions that are 
not identified separately in CALPHAD (for example, harmonic and non-harmonic vibrational contributions - which are both gathered in the excess Gibbs free energy, together with the non-ideal part of the configurational entropy). However it could easily be updated to take into account future improvement on that points. It could also be easily extended to Fe-Ni-Cr ternary alloys or to other binary or ternary systems. Finally the PIM is simple enough to be used as a basis for Atomistic Kinetic Monte Carlo methods to simulate the kinetics of homogeneous ordering or of heterogeneous precipitation of the $\mathrm{L}_{2}$ phase; or to model the interdiffusion properties in the $\gamma$ solid solution at high temperature. It is indeed not more numerically expensive than the PIM for BCC Fe-Cr alloys described in Ref. [47], which has been used for the simulations of precipitation kinetics [8, 9] or irradiation effects $[11,12]$. As in these studies, the modeling of kinetics will require the extension of the PIM to describe the formation and migration properties of point defects.

\section{ACKNOWLEDGMENTS}

We thank our colleagues Chu-Chun Fu and Maylise Nastar for many fruitful discussions. The research leading to these results has been carried out in the frame of EERA Joint Program for Nuclear Materials and is partly funded by the European Commission HORIZON 2020 Framework Programme under grant agreement No. 755269.

[1] C. Pareige, F. Soisson, G. Martin, and D. Blavette, Ordering and phase separation in Ni-Cr-Al: Monte carlo simulations vs three-dimensional atom probe, Acta Materialia 47, 1889 (1999).

[2] E. Clouet, M. Nastar, and C. Sigli, Nucleation of $\mathrm{Al}_{3} \mathrm{Zr}$ and $\mathrm{Al}_{3} \mathrm{Sc}$ in aluminum alloys: From kinetic monte carlo simulations to classical theory, Phys. Rev. B 69, 064109 (2004).

[3] E. Vincent, C. Becquart, and C. Domain, Solute interaction with point defects in $\alpha$-Fe during thermal ageing: A combined ab initio and atomic kinetic Monte Carlo approach, Journal of Nuclear Materials 351, 88 (2006), proceedings of the Symposium on Microstructural Processes in Irradiated Materials.

[4] M. Y. Lavrentiev, R. Drautz, D. Nguyen-Manh, T. P. C. Klaver, and S. L. Dudarev, Monte Carlo study of thermodynamic properties and clustering in the bcc Fe-Cr system, Phys. Rev. 
B 75, 014208 (2007).

[5] F. Soisson and C.-C. Fu, Cu-precipitation kinetics in $\alpha$-Fe from atomistic simulations: Vacancytrapping effects and Cu-cluster mobility, Phys. Rev. B 76, 214102 (2007).

[6] C. S. Becquart and C. Domain, Introducing chemistry in atomistic kinetic Monte Carlo simulations of fe alloys under irradiation, Physica Status Solidi (b) 247, 9 (2010).

[7] R. Ngayam-Happy, C. Becquart, C. Domain, and L. Malerba, Formation and evolution of $\mathrm{MnNi}$ clusters in neutron irradiated dilute fe alloys modelled by a first principle-based AKMC method, Journal of Nuclear Materials 426, 198 (2012).

[8] E. Martínez, O. Senninger, C.-C. Fu, and F. Soisson, Decomposition kinetics of Fe-Cr solid solutions during thermal aging, Phys. Rev. B 86, 224109 (2012).

[9] O. Senninger, E. Martínez, F. Soisson, M. Nastar, and Y. Bréchet, Atomistic simulations of the decomposition kinetics in Fe-Cr alloys: Influence of magnetism, Acta Materialia 73, 97 (2014).

[10] C. Liebscher, V. Radmilović, U. Dahmen, N. Vo, D. Dunand, M. Asta, and G. Ghosh, A hierarchical microstructure due to chemical ordering in the bcc lattice: Early stages of formation in a ferritic $\mathrm{Fe}-\mathrm{Al}-\mathrm{Cr}-\mathrm{Ni}-\mathrm{Ti}$ alloy, Acta Materialia 92, 220 (2015).

[11] O. Senninger, F. Soisson, E. Martinez, M. Nastar, C.-C. Fu, and Y. Brechet, Modeling radiation induced segregation in iron-chromium alloys, Acta Materialia 103, 1 (2016).

[12] F. Soisson, E. Meslin, and O. Tissot, Atomistic modeling of precipitation in fe-cr alloys under charged particles and neutron irradiations: Effects of ballistic mixing and sink densities, Journal of Nuclear Materials 508, 583 (2018).

[13] C. S. Becquart and F. Soisson, Monte carlo simulations of precipitation under irradiation, in Handbook of Mechanics of Materials, edited by S. Schmauder, C.-S. Chen, K. K. Chawla, N. Chawla, W. Chen, and Y. Kagawa (Springer Singapore, Singapore, 2019) pp. 703-731.

[14] I. V. Vernyhora, V. A. Tatarenko, and S. M. Bokoch, Thermodynamics of f.c.c. Ni-Fe Alloys in a Static Applied Magnetic Field, ISRN Thermodynamics 2012, 1 (2012).

[15] C. Yang, D. Williams, and J. I. Goldstein, A revision of the Fe-Ni phase diagram at low temperatures $\left(<400^{\circ} \mathrm{c}\right)$, Journal of Phase Equilibria 17, 522 (1996).

[16] Y. Mishin, M. Mehl, and D. Papaconstantopoulos, Phase stability in the Fe-Ni system: Investigation by first-principles calculations and atomistic simulations, Acta Materialia 53, 4029 (2005). 
[17] K. Li and C.-C. Fu, Ground-state properties and lattice-vibration effects of disordered Fe-Ni systems for phase stability predictions, Phys. Rev. Materials 4, 023606 (2020).

[18] G. Cacciamani, A. Dinsdale, M. Palumbo, and A. Pasturel, The Fe-Ni system: Thermodynamic modelling assisted by atomistic calculations, Intermetallics 18, 1148 (2010).

[19] I. Ohnuma, S. Shimenouchi, T. Omori, K. Ishida, and R. Kainuma, Experimental determination and thermodynamic evaluation of low-temperature phase equilibria in the $\mathrm{Fe}-\mathrm{Ni}$ binary system, Calphad 67, 101677 (2019).

[20] L. J. Swartzendruber, V. P. Itkin, and C. B. Alcock, The Fe-Ni (iron-nickel) system, Journal of Phase Equilibria 12, 288 (1991).

[21] J. Paulevé, D. Dautreppe, J. Laugier, and L. Néel, Une nouvelle transition ordre-désordre dans Fe-Ni (50-50 ), J. Phys. Radium 23, 841 (1962).

[22] A. Chamberod, J. Laugier, and J. Penisson, Electron irradiation effects on iron-nickel invar alloys, Journal of Magnetism and Magnetic Materials 10, 139 (1979).

[23] K. Reuter, D. B. Williams, and J. Goldstein, Ordering in the Fe-Ni system under electron irradiation, Metallurgical Transactions A 20, 711 (1989).

[24] K. Reuter, D. B. Williams, and J. Goldstein, Determination of the Fe- Ni phase diagram below $400^{\circ} \mathrm{C}$, Metallurgical Transactions A 20, 719 (1989).

[25] W. Xiong, H. Zhang, L. Vitos, and M. Selleby, Magnetic phase diagram of the Fe-Ni system, Acta Materialia 59, 521 (2011).

[26] T. Mohri, Y. Chen, and Y. Jufuku, First-principles calculation of L1 $1_{0}$-disorder phase equilibria for Fe-Ni system, Calphad 33, 244 (2009).

[27] P. J. Lawrence and P. L. Rossiter, Chemical and magnetic interactions in FCC Fe-Ni alloys using the cluster variation method, Journal of Physics F: Metal Physics 16, 543 (1986).

[28] M. B. Taylor, B. L. Gyorffy, and C. J. Walden, Magnetic and compositional order in nickel-rich $\mathrm{Ni}_{c} \mathrm{Fe}_{1-c}$ alloys, Journal of Physics: Condensed Matter 3, 1575 (1991).

[29] M.-Z. Dang and D. G. Rancourt, Simultaneous magnetic and chemical order-disorder phenomena in $\mathrm{Fe}_{3} \mathrm{Ni}, \mathrm{FeNi}$, and $\mathrm{FeNi}_{3}$, Phys. Rev. B 53, 2291 (1996).

[30] I. Vernyhora, D. Ledue, R. Patte, and H. Zapolsky, Monte carlo investigation of the correlation between magnetic and chemical ordering in nife alloys, Journal of Magnetism and Magnetic Materials 322, 2465 (2010).

[31] M. Taylor and B. Gyorffy, Monte carlo simulations of an fcc $\mathrm{Ni}_{c} \mathrm{Fe}_{1-c}$ alloy with vector magnetic 
freedom, Journal of Magnetism and Magnetic Materials 104-107, 877 (1992), proceedings of the International Conference on Magnetism, Part II.

[32] I. Vernyhora, H. Zapolsky, R. Patte, and D. Ledue, Atomic density function modeling of microstructure evolution in $\mathrm{Ni}_{3-x} \mathrm{Fe}_{x}$ alloys, Journal of Magnetism and Magnetic Materials 351, 52 (2014).

[33] M. Y. Lavrentiev, J. S. Wróbel, D. Nguyen-Manh, and S. L. Dudarev, Magnetic and thermodynamic properties of face-centered cubic fe-ni alloys, Phys. Chem. Chem. Phys. 16, 16049 (2014).

[34] J. S. Wróbel, D. Nguyen-Manh, M. Y. Lavrentiev, M. Muzyk, and S. L. Dudarev, Phase stability of ternary fcc and bcc Fe-Cr-Ni alloys, Phys. Rev. B 91, 024108 (2015).

[35] P. E. Blöchl, Projector augmented-wave method, Phys. Rev. B 50, 17953 (1994).

[36] G. Kresse and D. Joubert, From ultrasoft pseudopotentials to the projector augmented-wave method, Phys. Rev. B 59, 1758 (1999).

[37] G. Kresse and J. Hafner, Ab initio molecular dynamics for liquid metals, Phys. Rev. B 47, 558 (1993).

[38] G. Kresse and J. Furthmüller, Efficiency of ab-initio total energy calculations for metals and semiconductors using a plane-wave basis set, Comput. Mater. Sci. 6, 15 (1996).

[39] G. Kresse and J. Furthmüller, Efficient iterative schemes for ab initio total-energy calculations using a plane-wave basis set, Phys. Rev. B 54, 11169 (1996).

[40] J. P. Perdew, K. Burke, and M. Ernzerhof, Generalized Gradient Approximation Made Simple, Phys. Rev. Lett. 77, 3865 (1996).

[41] M. Methfessel and A. T. Paxton, High-precision sampling for Brillouin-zone integration in metals, Phys. Rev. B 40, 3616 (1989).

[42] H. J. Monkhorst and J. D. Pack, Special points for Brillouin-zone integrations, Phys. Rev. B 13, 5188 (1976).

[43] A. Zunger, S.-H. Wei, L. G. Ferreira, and J. E. Bernard, Special quasirandom structures, Phys. Rev. Lett. 65, 353 (1990).

[44] J. M. Cowley, An approximate theory of order in alloys, Phys. Rev. 77, 669 (1950).

[45] E. Martinez, C. C. Fu, M. Levesque, M. Nastar, and F. Soisson, Simulations of Decomposition Kinetics of Fe-Cr Solid Solutions during Thermal Aging, Solid State Phenom. 172-174, 1016 (2011). 
[46] M. Sansa, A. Dhouib, F. Ribeiro, B. Legrand, G. Tréglia, and C. Goyhenex, Tight-binding modelling of ferromagnetic metals and alloys, Modelling and Simulation in Materials Science and Engineering 25, 084004 (2017).

[47] M. Levesque, E. Martínez, C.-C. Fu, M. Nastar, and F. Soisson, Simple concentrationdependent pair interaction model for large-scale simulations of fe-cr alloys, Phys. Rev. B 84, 184205 (2011).

[48] M. Hillert, in Phase Equilibria, Phase Diagrams and Phase Transformations: Their Thermodynamic Basis (Cambridge University Press, 2007) Chap. 22, 2nd ed.

[49] U. Gahn, Ordering in face-centered cubic binary crystals confined to nearest-neighbour interactions - monte carlo calculations, Journal of Physics and Chemistry of Solids 47, 1153 (1986).

[50] G. Inden, Atomic ordering, in Phase Transformations in Materials, edited by G. Kostorz (VCH, 2001) Chap. 8, p. 519.

[51] E. V. Kozlov, A. S. Tailashev, and D. M. Shtern, Order-discharge transition in ni 3 fe alloy, Soviet Physics Journal 20, 583-588 (1977).

[52] E. Josso, Equilibrium Diagram for the Order to Disorder Transformation of Iron-Nickels near $\mathrm{Ni}_{3} \mathrm{Fe}$, C. R. Acad. Sci. 230, 1467 (1950).

[53] T. Heumann and G. Karsten, Karbonylverfahren und aufdampfverfahren zur bestimmung von phasengleichgewichten im temperaturbereich geringer beweglichkeit am beispiel der eisennickel-legierungen, Archiv für das Eisenhüttenwesen 34, 781 (1963).

[54] Van Deen, J. K. and Van Der Woude, F., Phase diagram of the order-disorder transition in ni3fe, J. Phys. Colloques 41, C1 (1980).

[55] J. V. deen] and F. V. D. Woude], Phase diagram of the order-disorder transition in ni3fe, Acta Metallurgica 29, 1255 (1981).

[56] Y. G. Chuang, Y. A. Chang, R. Schmid, and J. C. Lin, Magnetic contributions to the thermodynamic functions of alloys and the phase equilibria of Fe-Ni system below $1200 \mathrm{~K}$, Metallurgical Transactions A 17, 1361 (1986).

[57] G. Cacciamani, J. De Keyzer, R. Ferro, U. Klotz, J. Lacaze, and P. Wollants, Critical evaluation of the fe-ni, fe-ti and fe-ni-ti alloy systems, Intermetallics 14, 1312 (2006), eUROMAT 2005 'European Congress on Advanced Materials and Processes'. 